\title{
Cationized albumin-biocoatings for the immobilization of lipid vesicles ${ }^{\text {a) }}$
}

\author{
Sandra Ritz \\ Max Planck Institute for Polymer Research, Ackermannweg 10, D-55128 Mainz, Germany and Molecular \\ Biophysics, Johannes Gutenberg University, D-55128 Mainz, Germany \\ Klaus Eisele \\ Max Planck Institute for Polymer Research, Ackermannweg 10, D-55128 Mainz, Germany and Institute \\ of Organic Chemistry III, University of Ulm, Albert-Einstein-Allee 11, 89069 Ulm, Germany \\ Jan Dorn \\ Max Planck Institute for Polymer Research, Ackermannweg 10, D-55128 Mainz, Germany and Molecular \\ Biophysics, Johannes Gutenberg University, D-55128 Mainz, Germany \\ Shaohua Ding, Doris Vollmer, and Sabine Pütz \\ Max Planck Institute for Polymer Research, Ackermannweg 10, D-55128 Mainz, Germany \\ Tanja Weil \\ Max Planck Institute for Polymer Research, Ackermannweg 10, D-55128 Mainz, Germany; Institute \\ of Organic Chemistry III, University of Ulm, Albert-Einstein-Allee 11, 89069 Ulm, Germany; and Department \\ of Chemistry, National University of Singapore, 3 Science Drive, Singapore 117543, Republic of \\ Singapore \\ Eva-Kathrin Sinner ${ }^{\text {b) }}$ \\ Institute of Materials Research and Engineering (IMRE), 3 Research Link, 117602 Singapore, Republic \\ of Singapore and Molecular Biophysics, Johannes Gutenberg University, D-55128 Mainz, \\ Germany
}

(Received 9 July 2010; accepted 1 September 2010; published 14 December 2010)

\begin{abstract}
Tethered lipid membranes or immobilized lipid vesicles are frequently used as biomimetic systems. In this article, the authors presented a suitable method for efficient immobilization of lipid vesicles onto a broad range of surfaces, enabling analysis by quantitative methods even under rigid, mechanical conditions-bare surfaces such as hydrophilic glass surfaces as well as hydrophobic polymer slides or metal surfaces such as gold. The immobilization of vesicles was based on the electrostatic interaction of zwitterionic or negatively charged lipid vesicles with two types of cationic chemically modified bovine serum albumin (cBSA) blood plasma proteins (cBSA-113 and cBSA-147). Quantitative analysis of protein adsorption was performed as the cBSA coatings were characterized by atomic force microscopy, surface zeta potential measurement, fluorescence microscopy, and surface plasmon spectroscopy, revealing a maximal surface coverage $270-280 \mathrm{ng} / \mathrm{cm}^{2}$ for $0.02 \mathrm{mg} / \mathrm{ml} \mathrm{cBSA}$ on gold. Small unilamellar vesicles as well as giant unilamellar vesicles (GUVs) were readily immobilized ( $\sim 15 \mathrm{~min})$ on cBSA coated surfaces. GUVs with 5-10 mol\% negatively charged 1,2,-dipalmitoyl-sn-glycero-3-phosphoglycerol remained stable in liquid for at least 5 weeks. (C) 2010 American Vacuum Society. [DOI: 10.1116/1.3494039]
\end{abstract}

\section{INTRODUCTION}

The investigation of the role and function of membrane proteins such as protein receptors or ion-channels has been an area of high scientific interest due to their important contributions to many physiological processes and their implications in several diseases. ${ }^{1,2}$ Previously, membrane proteins have been investigated in native cell membranes; however, the cellular environment is highly complex and isolated molecular interactions are barely observed in such a "network situation." Considering the fact that roughly one-third of the native membranes consist of proteins, it is very likely that

\footnotetext{
This paper is part of an In Focus section on Biointerface Science in Singapore, sponsored by Bruker Optik Southeast Asia, IMRE, the Provost's Office and School of Materials Science and Engineering of Nanyang Technological University, and Analytical Technologies Pte. Ltd.

${ }^{\text {b) }}$ Author to whom correspondence should be addressed; electronic mail: sinner@mpip-mainz.mpg.de
}

other membrane proteins interfere with the membrane protein under investigation as well as its protein ligands or modulators.

Therefore, cell mimicking architectures, such as immobilized lipid vesicles (liposomes) containing a defined composition of proteins or protein complexes, offer an attractive alternative for studying membrane proteins in a molecular defined setting compared with the complexity of a cell. It has been shown that the three-dimensional, spherically closed lipid bilayer preserves the fluidity of a native cell membrane and could be used as a cell mimicking architecture acting as a reaction container when filled with cargoes such as proteins, ${ }^{3}$ DNA, ${ }^{4}$ or drug molecules for drug delivery. ${ }^{5}$

Immobilization of vesicles onto surfaces is an indispensable prerequisite for the use of vesicles as cellular mimicks allowing the application of various surface analytical techniques such as atomic force microscopy (AFM), surface 
plasmon spectroscopy (SPS), quartz crystal microbalance with dissipation, infrared spectroscopy, and microscopy.

Several immobilization strategies for lipid vesicles have been described, discriminating between nonspecific surface interactions driven by van der Waals and electrostatic forces, ${ }^{6,7}$ steric entrapment, ${ }^{8}$ and specific interactions mediated by two different binding partners such as biotin/streptavidin, ${ }^{9,10}$ single stranded DNA and the complementary DNA strand, ${ }^{11,12}$ as well as histidine/nickel and nitrilotriacetic acid. ${ }^{13,14}$ The single stranded DNA approach even allowed the addressable immobilization of lipid vesicles. ${ }^{15}$ A comprehensive overview over all these techniques was published recently. ${ }^{16}$ Most examples of these pioneering methods have been described for the attachment of small unilamellar vesicles (SUVs) $(<200 \mathrm{~nm})$ or large unilamellar vesicles and much less for giant unilamellar vesicles (GUVs) $(10-100 \mu \mathrm{m})$.

However, GUVs are particularly attractive as cell mimicking entities containing a simplified lipid/protein composition. GUVs can be visualized by standard microscopy techniques, facilitating the observation of vesicular-ensembles for sensing and screening applications. Additionally, GUVs are suitable architectures for single vesicle experiments that allow an improved understanding of, e.g., domain assembly, membrane curvature ${ }^{17}$ or protein/receptor interactions. ${ }^{18}$

One major limitation of immobilized GUVs is restoring their stability over longer time periods and keeping the delicate balance between mere adhesion of intact vesicles onto a surface and the phenomenon of complete vesicle spreading into planar membrane architecture. Positively charged polylysine (PLL) coatings have been successfully used for the stabilization and embedding of SUVs in polyelectrolyte multilayers. ${ }^{19}$ However, a PLL coated surface was also described to result in strong surface adhesion and subsequent fusion of GUV vesicles. ${ }^{20}$ Bovine serum albumin (BSA), a commonly used surface blocking agent in biosensor applications, revealed only a weak adherence to giant vesicles made out of stearoyl-oleoyl-phosphatidylcholine ${ }^{21}$ or palmitoyl-oleoyl-phosphatidylcholine. ${ }^{22}$

Giant vesicles were successfully immobilized by a combination of BSA coating and picket fences of the polymer polyimide $^{22}$ or by specific receptor-ligand interaction such as lipid-anchored sialyl-Lewis ${ }^{\mathrm{x}}$ molecules with surface immobilized E-selectin. ${ }^{18}$ Liu et al. formed a GUV microarray on glass supports by controlled dehydration-rehydration, and the GUVs where stable for a few hours. ${ }^{23}$

In this article, we will present a more facile way for the efficient and long time immobilization of lipid vesicles (SUVs-GUVs) on bare surfaces such as hydrophilic glass surfaces as well as hydrophobic polymeric flow channels [cyclo olefine polymer (COP)/cyclo olefine copolymer $(\mathrm{COC})]$ and gold. We used two different chemically modified BSA proteins with positive net charges (cBSA), cBSA-147 and cBSA-113, as universal coatings for the different substrates and for the immobilization of SVUs and GVUs. The liposomes partly fused on the cBSA coated supports, suggesting a mixed model of supported lipid bilayers and immobilized vesicles.

Cationized BSA protein material is achieved by reacting accessible carboxylic acid groups with ethylenediamine, thus leading to a protein mixture with a random number and distribution of amino groups and positive net charges. Such protein materials have been applied as a highly immunogenic carrier protein for preparing antigens for antibody production. Recently, in a series of chemically modified BSA species with narrow distributions, more distinct numbers of primary amino groups have been prepared and investigated regarding their potential to interact with negatively charged DNA. ${ }^{24,25}$ Only the two highest cationic albumin protein fractions, cBSA-113 and cBSA-147 with an average of 113 and 147 primary amino groups $(\mathrm{pI}>11)$, respectively, were found to interact tightly with DNA. Therefore, these two protein species have been considered as cationic biocoatings for the immobilization of cells ${ }^{25}$ or negatively charged liposomes as described herein.

Our approach offers an alternative and straightforward tethering method for lipid vesicles and supported lipid bilayers, where the modified cBSA serves as a biocompatible spacer separating the lipids from direct substrate interaction.

\section{MATERIALS AND METHODS}

\section{A. Materials and chemicals}

Native BSA (fraction V), 1,2-dioleoyl-sn-glycero-3phosphoethanolamine (DOPE), cholesterol $(\mathrm{CH})$, chloroform (high pressure liquid chromatography (HPLC) grade), and ethanol (HPLC grade) were obtained from Sigma Aldrich (Germany). 1,2-dioleoyl-sn-glycero-3-phosphocholine (DOPC) and 1,2,-dipalmitoyl-sn-glycero-3-phosphoglycerol (DPPG) was obtained from Avanti Polar Lipids (Alabama, USA). The lipids were dissolved in chloroform (HPLC grade) and kept as $10 \mathrm{mM}$ stock solutions prior to mixing. All cleaning procedures for glass were performed with $2 \%$ Hellmanex II (Helma, Germany) diluted in ultrapure water. BSA and SUVs were prepared in a Dulbecco's phosphate buffered saline (DPBS) buffer $(2.7 \mathrm{mM} \mathrm{KCl}, 1.5 \mathrm{mM}$ $\mathrm{KH}_{2} \mathrm{PO}_{4}, 138 \mathrm{mM} \mathrm{NaCl}, 8 \mathrm{mM} \mathrm{Na}_{2} \mathrm{HPO}_{4}$, Gibco/Invitrogen, Germany). All aqueous solutions were prepared in ultrapure water (18.2 $\mathrm{M} \Omega / \mathrm{cm}, 0.22 \mu \mathrm{m}$ filter, Millipore, Germany).

\section{B. Preparation of cBSA coated surfaces}

The glass coverslips $\left(20 \times 20 \times 0.17 \mathrm{~mm}^{3}\right.$, Menzel, Germany) and eight-well chambers with glass bottom (Lab-Tek, Nunc, Germany) were cleaned by sonication for 15 min with a $2 \%$ Hellmanex solution in an ultrasound bath, washed carefully with ultrapure water, sonicated again for $15 \mathrm{~min}$ in a $2 \%$ Hellmanex solution, washed again carefully with water, and then finally sonicated for $15 \mathrm{~min}$ in absolute ethanol. The coverslips were dried under a stream of nitrogen and used directly. The polymer slides ( $\mu$-slide VI, uncoated, Ibidi, Germany) were delivered in a sterile packing and used without further cleaning. The planar gold surfaces were prepared 
on LaSFN9 glass substrates $(n=1.845$ at $\lambda=632.8 \mathrm{~nm}$, Berlinger Glas, Germany) by evaporating $99.99 \%$ gold to a final thickness of $\sim 50 \mathrm{~nm}$ in an evaporating system using an electron gun (Edwards FL400, Boc Edwards, UK). The gold slides were stored in glass containers under argon prior to use.

The preparation and characterization of cationized BSA, cBSA-113 and cBSA-147, as well as cBSA-147 labeled with the fluorescent chromophore tetramethylrhodamine (cBSA147-TMR) for fluorescence imaging studies have been reported before. $^{24,25}$ Native BSA, cBSA-113, cBSA-147-TMR, or cBSA-1147-TMR was dissolved as $2 \mathrm{mg} / \mathrm{ml}$ solution in sterile DPBS buffer and stored for up to 4 weeks at $4{ }^{\circ} \mathrm{C}$. For the BSA coating, different kinds of cleaned substrates (glass, polymer, and gold) were treated for $30 \mathrm{~min}$ with the indicated concentrations of the individual BSA species at room temperature, and then rinsed 5-10 times with DPBS.

\section{Preparation of SUVs}

A lipid mixture of DOPC/DOPE/CH/DPPG $(40 / 25 / 30 / 5 \mathrm{~mol} \%)$ or DOPC/DPPG $(95 / 5 \mathrm{~mol} \%)$ was prepared from $10 \mathrm{mM}$ stock solutions in chloroform and $500 \mu \mathrm{l}$ were pipetted into a $50 \mathrm{ml}$ glass vial. The solvent was evaporated under a stream of nitrogen, and the lipid film was dried for at least $2 \mathrm{~h}$ under vacuum $\left(1 \times 10^{-2}\right.$ bar $)$. The lipid film was rehydrated as a multilamellar vesicle in $5 \mathrm{ml}$ DPBS buffer $(2 \mathrm{mM})$ by three cycles of vortexing, freezing in liquid nitrogen, and thawing at $40{ }^{\circ} \mathrm{C}$ in a water bath. Aliquots were stored at $-20{ }^{\circ} \mathrm{C}$ until use.

SUVs were prepared by extruding multilamellar liposomes 21 times through a polycarbonate membrane $(50 \mathrm{~nm}$ pore size, Avestin, Ottawa, Canada) with a hand held vesicle extruder (LipoFast, Avestin). Extruded SUVs were directly applied on the surface preventing inner vesicular fusion.

\section{Preparation of GUVs}

Giant vesicles were prepared by following the electric swelling method. ${ }^{26,27}$ Briefly, $25 \mu \mathrm{l}$ of a $10 \mathrm{mM}$ lipid mixture dissolved in chloroform was introduced dropwise onto indium tin oxide (ITO) coated glass electrodes $\left(5 \times 5 \mathrm{~cm}^{2}\right.$, $20 \mathrm{~nm}$ ITO) and dried under vacuum for $2 \mathrm{~h}$. The two electrodes were clamped together and separated by a PDMS spacer of $1 \mathrm{~mm}$ thickness. The gap was filled with $400 \mu \mathrm{l}$ of $200 \mathrm{mM}$ sucrose, and the electrodes were connected to an ac AC voltage generator $(20 \mathrm{MHz}$ programmable function generator, Series 8200, Kontron Messtechnik, Germany) under the following conditions: $1 \mathrm{~V}$ at $10 \mathrm{~Hz}$ for $2 \mathrm{~h}$ followed by 1 $\mathrm{V}$ at $5 \mathrm{~Hz}$ for $30 \mathrm{~min}$. Zwitterionic GUVs (DOPC, DOPE, $\mathrm{CH}, 45: 25: 30 \mathrm{~mol} \%$ ) or negatively charged GUVs (DOPC, DOPE, CH, DPPG, 40:25:30:5 mol \%) were diluted in the DPBS buffer $(1: 20)$ and incubated for $15 \mathrm{~min}$ on the coated support (see Sec. II B) containing the respective modified BSA species.

\section{E. Microscopic characterization}

Time laps imaging (movies) and fluorescence imaging of attached vesicles were performed on a wide field IX70 microscope (Olympus, Germany) equipped with a mercury lamp, $20 \times$ LCP Plan Fl air objective, emission filter cube for rhodamine (ex. 530-550 nm/dichroic mirror 570/em. 590 $\mathrm{nm}$ ), and a color charge-coupled device camera from Soft Imaging System (Germany). Image processing was done with Cell D 3.3 (Olympus) and IMAGEJ (NIH, freeware).

\section{F. Fluorescence recovery after photobleaching measurements}

Lipid surfaces were prepared in chamber slides with glass or polymer bottom and were labeled for fluorescence microscopy with the lipophilic tracer DiD (ex. $644 \mathrm{~nm}$, em. 665, Invitrogen, Darmstadt, Germany). $3 \mathrm{mM}$ DiD oil solution dissolved in DMSO was diluted to a final concentration of $1.5 \mathrm{nM}$ DiD $(\sim 0.005 \mathrm{~mol} \%)$ in DPBS, added to the prepared lipid surfaces, and incubated for $15 \mathrm{~min}$ followed by rinsing with DPBS. Measurements were performed on a Leica TCS SP laser scanning microscope (Leica, Germany) equipped with a $10 \mathrm{~mW}, 633 \mathrm{~nm}$ HeNe laser, a 63 $\times 1.4$ NA oil Plan Apo CS objective, and a photomultiplier with an emission detection range set to $650-750 \mathrm{~nm}$. The image size was $82 \times 82 \mu \mathrm{m}^{2}$ and the scanning frequency was $1000 \mathrm{~Hz}$. The following bleaching procedure with a time of 0.278 s/frame was used: 20 frames prebleaching with $10 \%$ laser intensity, 50 frames bleaching with $100 \%$ laser intensity, and 300 frames postbleaching with $10 \%$ laser intensity. Bleaching was done with a square-well beam, with a bleaching spot diameter of 6 or $14 \mu \mathrm{m}$, having uniform bleach intensity over a disk. In order to compensate for photobleaching through imaging, the actual intensity $I_{\text {frap }}$ was normalized by an unbleached area of the same size $I_{\text {ref }}$ like the bleached area for every time point as follows:

$$
I_{\text {norm }}(t)=I_{\text {frap }}(t) / I_{\text {ref }}(t) .
$$

The parameters $y_{o}$ (final value for the curve fitting on the $y$-axis), $A$ (level of fluorescence recovery after photobleaching), $\tau$ (time constant), $t_{1 / 2}$ (half-time of fluorescence recovery), and $M$ (mobile fraction) were estimated by a single exponential function and nonlinear curve fitting (LevenbergMarquardt algorithm) using the Leica fluorescence recovery after photobleaching (FRAP) wizard based on the following equations:

$$
\begin{aligned}
& F(t)=y_{o}-A^{*} e^{-t / \tau}, \\
& t_{1 / 2}=\ln 2 * \tau, \\
& M=\frac{F_{\infty}-F_{0}}{F_{i}-F_{0}},
\end{aligned}
$$

where $F_{\infty}$ is the fluorescence after recovery, $F_{o}$ is the fluorescence right after bleaching, and $F_{i}$ is the fluorescence before bleaching. 


\section{G. Atomic force microscopy}

Cationized BSA-113 coated glass coverslips $(20 \times 20$ $\times 0.17 \mathrm{~mm}^{3}$, Menzel, Germany) and cBSA-113 coated gold substrates were directly investigated after washing with DPBS in a liquid environment by an AFM (Nanowizard, JPK Instruments, Berlin, Germany), installed on an inverted microscope (Axiovert 200, Zeiss, Germany). Images were recorded in the soft tapping mode (setpoint: 90\%-95\% of the lock-in amplitude) with an oxide sharpened silicon nitride tip (NP-S, $0.32 \mathrm{~N} / \mathrm{m}$ nominal spring constant, Veeco, Mannheim, Germany). No special cleaning procedure for the cantilever was applied. Images were recorded at resonance frequencies between 9 and $12 \mathrm{kHz}$ and a scanning speed of 1 Hz. Finally, all images were processed with the GWYDDION 2.14 software. Roughness values (rms) were calculated from $1 \mu \mathrm{m}^{2}$ images with a resolution of $512 \times 512$ pixels.

\section{H. Surface plasmon spectroscopy}

The adsorption of cBSA-113 on gold was monitored by SPS. The measurements were performed in a home-built apparatus $^{28}$ (RT-2005, ResTec, Germany) equipped with a HeNe laser $(633 \mathrm{~nm})$ and a glass flow cell for liquid handling ( $\sim 20 \mu$ l volume, Helma, Germany). The evaporated gold slide (LaSFN9) was set on the glass flow cell and mounted on a glass prism (LaSFN9) in Kretschmann configuration with an index matching oil $(n=1.7$, Cargille Laboratory., Cleveland, $\mathrm{OH}$ ). The obtained data were monitored using the WASPLAS software written by Andreas Scheller (Max Planck Institute for Polymer Research, Mainz). All experiments were performed in PBS with a flow rate of $400 \mu \mathrm{l} / \mathrm{min}$. Surface plasmons can be resonantly excited at a metal/ dielectric interface by a $p$-polarized monochromatic light source only at a well-defined angle of incidence (resonance angle). This is strongly dependent on the refractive index (or dielectric constant) profile of the samples within the evanescent field. When molecules adsorb to the sensor surface, a change in the refractive index close to the surface occurs. The corresponding shift in the resonance angle $(\Delta \theta)$ is proportional to the surface concentration of the molecule expressed as optical thickness $(d \cdot n)$, where $d$ is the geometrical thickness of the layer and $n$ is the refractive index of the dielectric. The optical thickness was converted into the geometrical thickness by curve fitting with WINSPALL software based on the Fresnel equation and transfer matrix algorithm (3.01, Max Planck Institute for Polymer Research, Mainz).

\section{Surface zeta potential}

The electroosmotic flow profile of standard beads was measured in a flat surface cell unit (Beckmann Coulter, Krefeld, Germany) with a Delsa Nano C device (Beckmann Coulter) and calculated into surface zeta potential by using the Smoluchowski equation. ${ }^{29}$ After cell assembly, the cell constant was measured in $10 \mathrm{mM} \mathrm{NaCl}\left(67 \pm 3 \mathrm{~cm}^{-1}\right)$, verifying correct assembly without air bubbles. Afterward the cell was rinsed with ultrapure water and filled with standard polystyrene latex particles (Otsuka Electronics, Japan) diluted 1:100 in PBS. Measurements have been conducted according to the manufacturer's instruction.

\section{J. Contact angle measurement}

Contact angle measurements were performed applying the static sessile drop method with a fully computer-controlled instrument (Krüss, Germany). The measurements were carried out under constant ambient conditions and a constant drop size $(3 \mu l)$. Ultrapure water was used as medium.

\section{RESULTS AND DISCUSSION}

\section{A. Coating of hydrophilic and hydrophobic surfaces with cBSA-113 and cBSA-147}

The adsorption of native BSA on various surfaces represents a well-established process since several different coating and blocking conditions have been described before with concentrations ranging from 0.005 to $50 \mathrm{mg} / \mathrm{ml}$ and incubation times from $10 \mathrm{~min}$ to $2 \mathrm{~h}^{30} \mathrm{BSA}$ represents a globular soft protein, which can undergo structural and conformational changes. Two adsorption mechanisms of BSA, e.g., the side-on or the end-on adsorption with the major axes horizontal or perpendicular to the surface, have been discussed in the literature. ${ }^{31-33}$ However, the formation of dimers, multilayers, or aggregates on the first, mostly irreversible bound, monolayer has also been observed. ${ }^{34}$ Important factors that influence the architecture of the surface coating include the surface material, the concentration, the incubation time, and the buffer conditions. Herein, a phosphate buffer was chosen which allows protein adsorption in a more compact and stable conformation due to the high binding energy of phosphate and BSA. ${ }^{35}$

We present here the adsorption of GUVs onto cBSA-113, cBSA-147, and native BSA using various substrates. In contrast to native BSA, both cBSA species contain a high density of primary amino groups, which are positively charged at physiological $p \mathrm{H}$. In order to elucidate the potential of cBSA-113 and cBSA-147 as universal coating biomaterials, different surfaces such as hydrophilic and negatively charged glass surfaces, hydrophobic evaporated gold films $(50 \mathrm{~nm})$, and commercial optical transparent polymer slides (COP/ COC) were applied.

\section{Glass surface coating}

The adsorption of different concentrations of cBSA-147 on glass surfaces has been investigated previously by applying AFM. cBSA-147 monolayers with vertical heights of 5-6 nm were formed. ${ }^{25}$ Cationized BSA-113 also readily adsorbs on glass and Fig. 1(a) represents a typical AFM height image of adsorbed cBSA-113 $(0.1 \mathrm{mg} / \mathrm{ml})$ by liquid AFM measurements. The cross section revealed a valley to peak height of 2-5 $\mathrm{nm}$, and only a few bigger aggregates $(5-30 \mathrm{~nm})$ were detected. There was no significant difference of the surface roughness pattern in the chosen concentration range $(0.025$, $0.5,0.1,1 \mathrm{mg} / \mathrm{ml})$. The $\mathrm{rms}$ roughness was around $1 \mathrm{~nm}$. A 

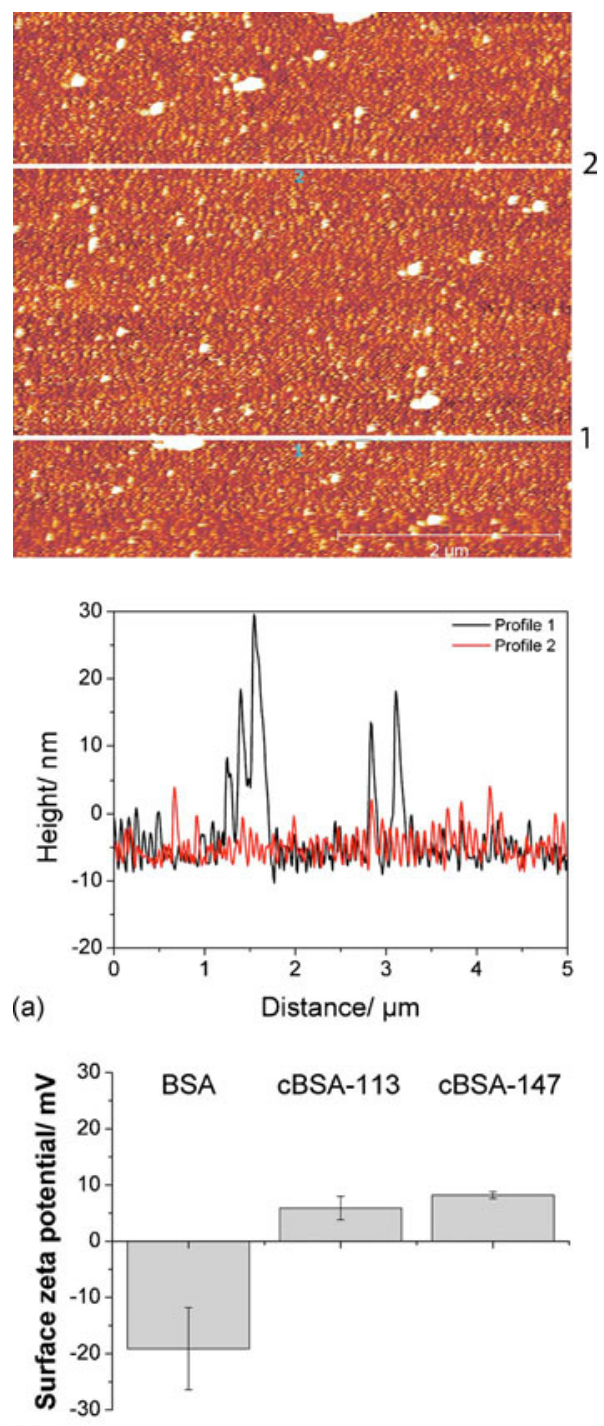

(b)

FIG. 1. (Color online) Adsorption of cBSA-113 on glass surface. (a) AFM height image of $0.1 \mathrm{mg} / \mathrm{ml}$ cBSA-113 with horizontal profiles. (b) Surface zeta potential of native BSA, c-BSA-113, and cBSA-147. Mean value and standard deviation calculated from two independent experiments $(n=6)$.

slightly increased roughness (rms) of $1.35 \pm 0.03 \mathrm{~nm}$ was observed only at low concentrations $(25 \mu \mathrm{g} / \mathrm{ml})$.

Mass spectra analysis revealed an average number of amino groups of 60 for native BSA, which results in a calculated net charge of $-16 \mathrm{mV}(p \mathrm{H}$ 7.4). The cationized BSA carries an average number of 113 or 147 amino groups, which results in a calculated positive net charge of $+90 \mathrm{mV}$

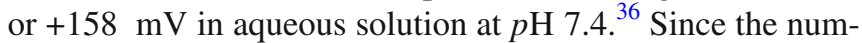
ber of charges within the protein scaffold strongly depends on the buffer conditions ( $p \mathrm{H}$, ionic strength), the surface zeta potential of adsorbed BSA in ultrapure water (see Ref. 37 for Fig. 8) and DPBS buffer [Fig. 1(b)] was determined by the light scattering of the electroosmotic flow of polystyrene beads in a flow chamber where one side of the channel was provided by the sample. Native BSA revealed a net negative charge in DPBS of $-19 \pm 7 \mathrm{mV}$, whereas cBSA-113 and cBSA-147 were positively charged and different positive net charges of $+6 \pm 2 \mathrm{mV}$ for cBSA-113 and $+8.2 \pm 1 \mathrm{mV}$ for cBSA-147 were found, reflecting the higher degree of cationization of cBSA-147 versus cBSA-113. ${ }^{25}$ Glass acquires a negative surface charge density, primarily due to the dissociation of terminal silanol groups in water. ${ }^{38}$ The cBSA coated glass has a positive net charge in the given buffer system. Although the estimated Debye-Hückle length is less than $1 \mathrm{~nm}(\sim 0.82 \mathrm{~nm}$ at a concentration of $138 \mathrm{mM}$ $\mathrm{NaCl} / 25^{\circ} \mathrm{C}$; the other buffer components were neglected for the calculation of the Debye-Hückle length), opposite electrostatic charges probably have a stronger impact on the adsorption process of cationic BSA than on native BSA.

\section{Polymer surface coating}

To verify the adsorption of cBSA species on polymer slides, different concentrations $(0.01,0.1$, and $1 \mathrm{mg} / \mathrm{ml}$, PBS, $p \mathrm{H}$ 7.4) of tetramethylrhodamine labeled native and cBSA147 were tested and analyzed by fluorescence microscopy with an emission filter at $590 \mathrm{~nm}$. At all concentrations, a pronounced fluorescence signal was found, which decreased at lower protein concentrations (data not shown). The polymer slide $(\mathrm{COP} / \mathrm{COC})$ is characterized by a negative surface zeta potential of $\sim-40 \mathrm{mV}(10 \mathrm{mM} \mathrm{NaCl})$ and a surface tension of $\sim 36 \mathrm{mN} / \mathrm{m} .{ }^{39}$ For a direct comparison of the surfaces used here, we measured the contact angle to water. The polymer surface exhibited a contact angle of $77^{\circ}$. This suggests an adsorption mechanism based on hydrophobic interaction with nonpolar side groups.

\section{Gold surface coating}

For both native and cationic BSA species, interactions with the gold surface were found. SPS analysis of the adsorption kinetic of different cBSA-113 concentrations $(0.002-0.2 \mathrm{mg} / \mathrm{ml}, \mathrm{PBS})$ on evaporated gold is shown in Fig. 2(a). The adsorption kinetic is expressed as distance change versus the time at a fixed angle of incidence. The highest change in reflectivity (distance) was obtained for $0.02 \mathrm{mg} / \mathrm{ml}$ cBSA-113 [Fig. 2(b)], resulting in an average layer thickness of $7.3 \pm 2 \mathrm{~nm}$ as determined by curve fitting of the SPRscanning curves from three independent experiments [Fig. 2(c)]. The surface coverage of the cBSA was calculated as described $^{40}(\Gamma=d c / d n \cdot \Delta n \cdot d)$ and resulted in a surface coverage of $270-280 \mathrm{ng} / \mathrm{cm}^{2}$, using refractive indices of $n_{\text {DPBS }}=1.33$ and $n_{\text {Protein }}=1.4{ }^{28}$ It should be mentioned that the fitting process considered a uniform film thickness as boundary condition. Figure 2(d) shows the AFM height image of adsorbed cBSA-113 on gold. The rms was determined from an area of $1 \mu \mathrm{m}^{2}$ and is around $1.4 \mathrm{~nm}$, and the topography is similar to the AFM data for the adsorption of cBSA113 on glass. The cBSA-coating is uniform with some attached aggregates on top. The AFM images prove that a uniform coverage can be assumed.

Human bovine serum albumin, which has a high percentage of sequence identities, ${ }^{34}$ has a molecule size of 5.5 $\times 5.5 \times 9 \mathrm{~nm}^{3}$. This means that only one protein layer is present. 

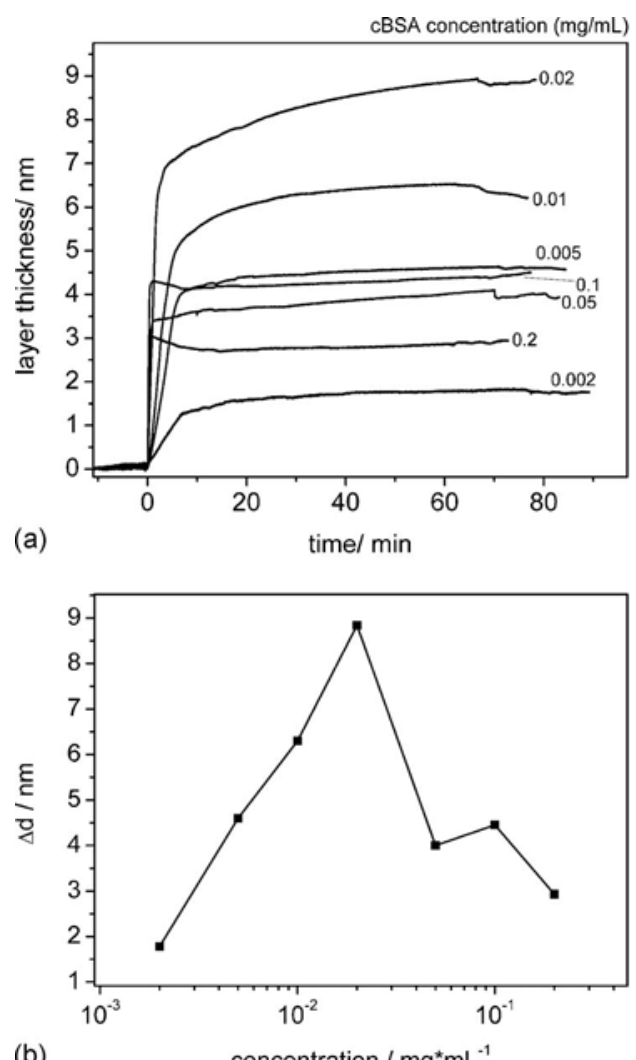

(b)

concentration $/ \mathrm{mg}^{*} \mathrm{~mL}^{-1}$
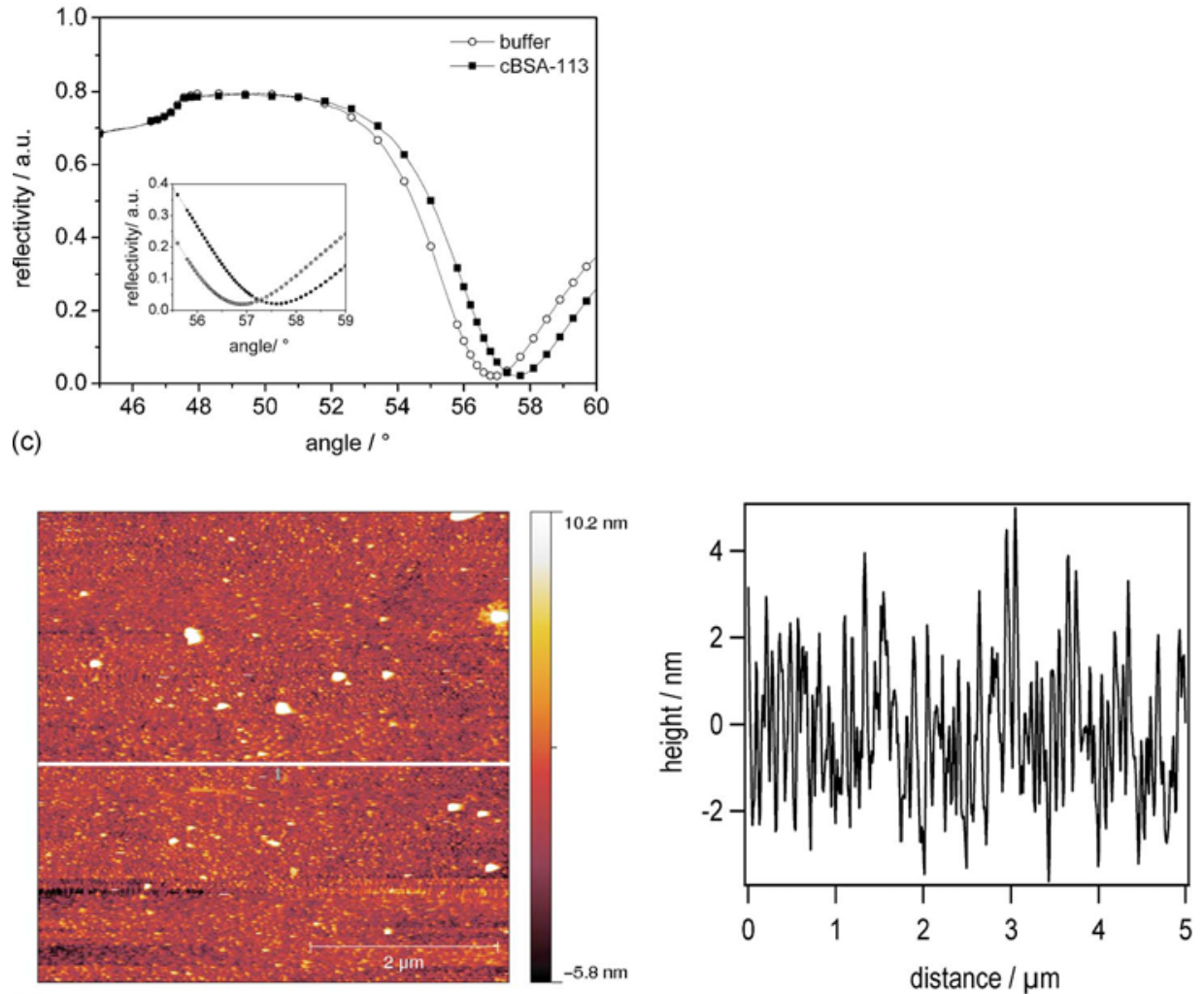

(d)

FIG. 2. (Color online) Adsorption of cationic BSA on gold. (a) SPS kinetic of concentration dependent adsorption of cBSA-113 (0.002-0.2 mg/ml) on gold. (b) Layer thickness dependence of cBSA-113 concentration extracted from the SPR kinetic. (c) SPR angle scan of buffer DPBS and cBSA-113 (0.02 mg/ml). (e) AFM height image of cBSA-113 on gold $(0.025 \mu \mathrm{g} / \mathrm{ml})$ with a horizontal profile below. 


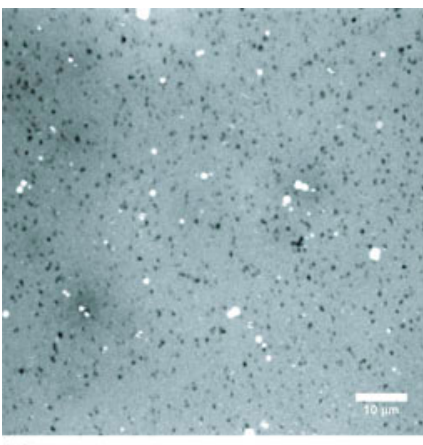

(a)

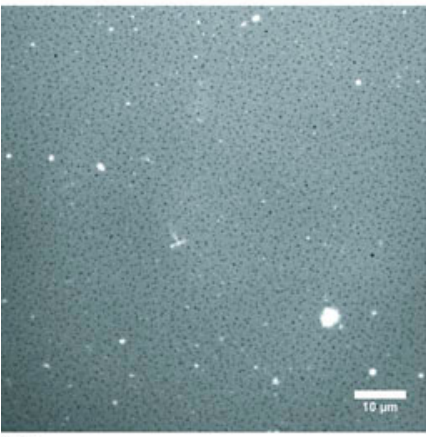

(e)

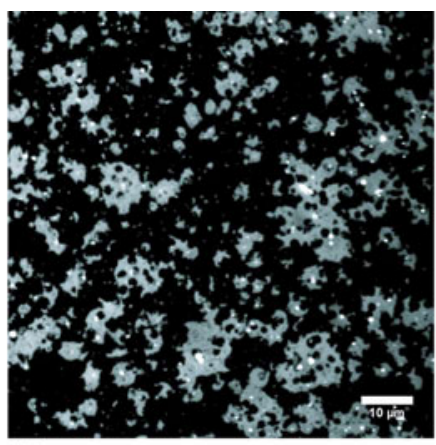

(b)

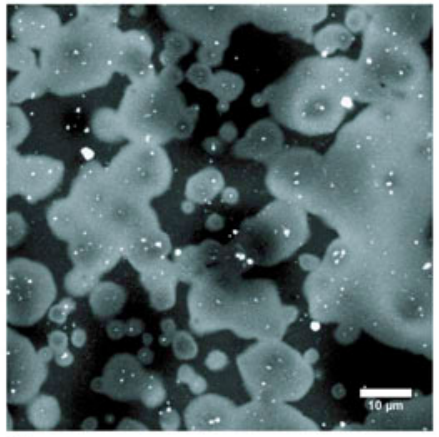

(f)

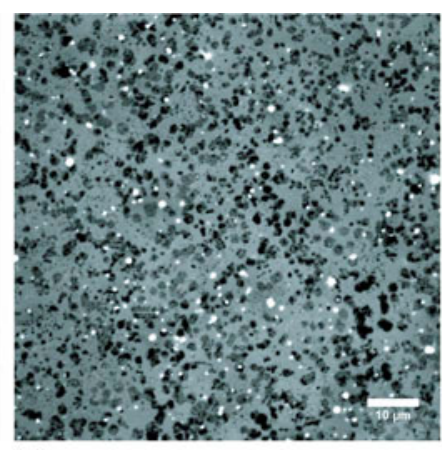

(c)

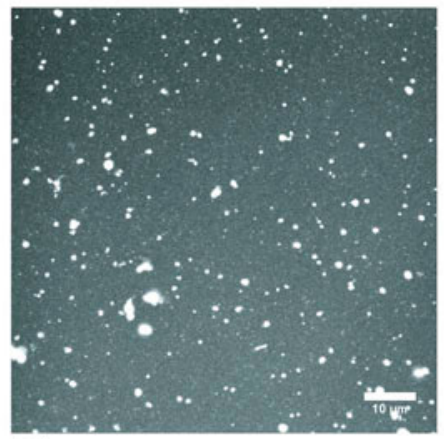

(g)

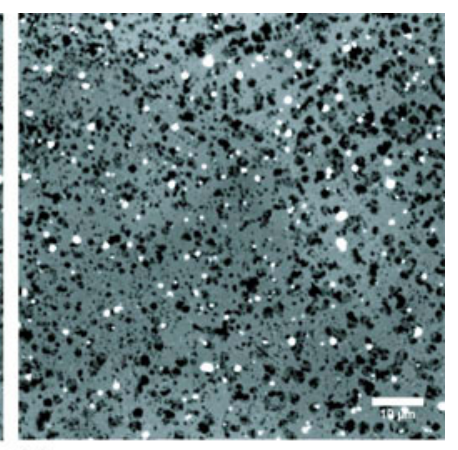

(d)

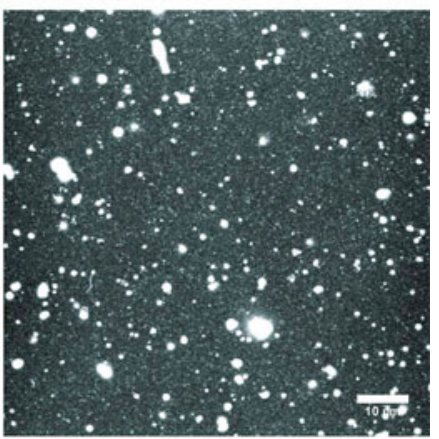

(h)

Fig. 3. (Color online) Fluorescent confocal microscopy images of lipid layers formed by the fusion of small vesicles (DOPC/DPPG, $95 / 5 \mathrm{~mol} \%$ ) on glass [(a)-(d)] and polymer [(e)-(h)] chamber slides without coating [(a) and (e)] or coated with $0.1 \mathrm{mg} / \mathrm{ml}$ native BSA [(b) and (f)], cationic BSA-113 [(c) and (g)], and cationic BSA-147 [(d) and (h)]. Lipids were fluorescently stained with DiD. The bright fluorescent spots were adsorbed SUV on the top of the lipid bilayer. Bar is scaled to $10 \mu \mathrm{m}$. (a) Lipid bilayer on glass. (b) Lipid patches on glass coated with nBSA. Lipid bilayer network on glass coated with (c) cBSA-113 or (d) cBSA-147. (e) Lipid monolayer on polymer. (f) Lipid patches on polymer coated with nBSA. Lipid bilayer on polymer coated with (g) cBSA-113 or (h) cBSA-147.

According to the results described above, the driving force for the adhesion of cBSA species on negatively charged hydrophilic surfaces (contact angle too small to be measured), such as glass, may be the electrostatic interactions between the surface and the protonated amino groups, besides van der Waals and hydrophobic interactions. On hydrophobic surfaces like the polymer slides applied herein, the nonpolar side chains of the protein are most likely responsible for the interaction. On gold, which is moderately hydrophobic (contact angle $88^{\circ}$ ), both native and the cBSA species could be attached most likely by hydrophobic interactions as well as due to the presence of functional groups that can interact with gold such as cysteine residues (BSA contains one free cysteine and 17 intrachain disulfide bounds) allowing covalent interactions. ${ }^{41} \mathrm{~A}$ maximal surface coverage at a concentration of $0.02 \mathrm{mg} / \mathrm{ml}$ on gold of $270-280 \mathrm{ng} / \mathrm{cm}^{2}$ was calculated from the SPS kinetic. This result indicated that (i) higher BSA concentrations might not lead to increased mass adsorptions. This observation is in agreement with previous results where a time and concentration dependent adsorption model for native BSA on hydrophobic polyethylene surfaces and a critical concentration (1000 ppm, $0.06 \mathrm{mg} / \mathrm{ml}$ ) were found. ${ }^{33}$ BSA adsorption below the critical concentration proceeds in a normal side-on mechanism, whereas for higher concentrations, the adsorption proceeds in a crowded or aggregated way, which is similar to end-on, but with gaps between the adsorbed molecules.
Using the protein size mentioned above, the maximum surface coverage is $231 \mathrm{ng} / \mathrm{cm}^{2}$ for a side-on orientation and $386 \mathrm{ng} / \mathrm{cm}^{2}$ for an end-on orientation. Based on the SPS kinetic results for both cBSA species $\left(270-280 \mathrm{ng} / \mathrm{cm}^{2}\right)$, we (ii) suggest the formation of a mixed side-on and end-on cBSA monolayer with an approximate thickness of about 7.3 nm.

\section{B. Immobilization of SUV on CBSA coated polymer and glass slides}

Fluorescence microscopy imaging (Fig. 3) and FRAP (Table I) measurements were performed to study the adsorp-

TABLE I. FRAP measurements of the lipophilic dye DiD as tracer for the lipid bilayer fluidity on cationized BSA coated glass and polymer chamber slides (COP/COC).

\begin{tabular}{lcccc}
\hline \hline Support & Surface coating & $\begin{array}{c}\text { Surface } \\
\text { coverage } \\
(\%)\end{array}$ & $\begin{array}{c}\text { Mobile } \\
\text { fraction } \\
(\%)\end{array}$ & $\begin{array}{c}t_{1 / 2}(\mathrm{~s}) \\
\text { (half time of } 50 \% \\
\text { fluorescence recovery) }\end{array}$ \\
\hline Glass & $\ldots$ & 100 & $88 \pm 1$ & $4.9 \pm 0.8$ \\
Glass & cBSA-113 & $76 \pm 5$ & $72 \pm 6$ & $12.5 \pm 4$ \\
Glass & cBSA-147 & $87 \pm 5$ & $67 \pm 3$ & $13.1 \pm 1$ \\
COP/COC & $\ldots$ & 100 & $84 \pm 2$ & $14.6 \pm 0.8$ \\
COP/COC & cBSA-113 & 100 & $70 \pm 4$ & $20.5 \pm 0.4$ \\
COP/COC & cBSA-147 & 100 & $60 \pm 1$ & $16.8 \pm 2.7$ \\
\hline \hline
\end{tabular}




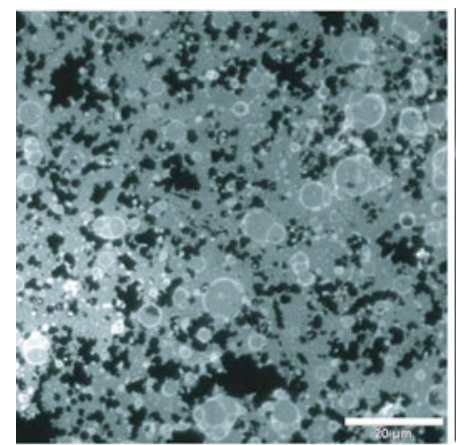

(a)

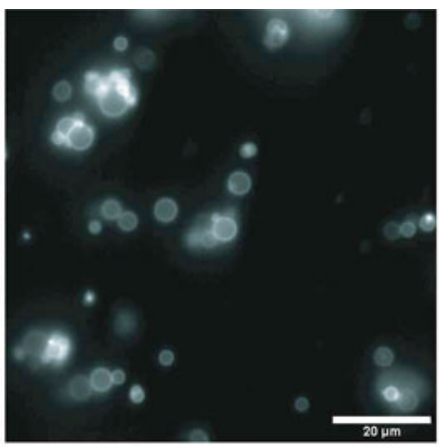

(b)

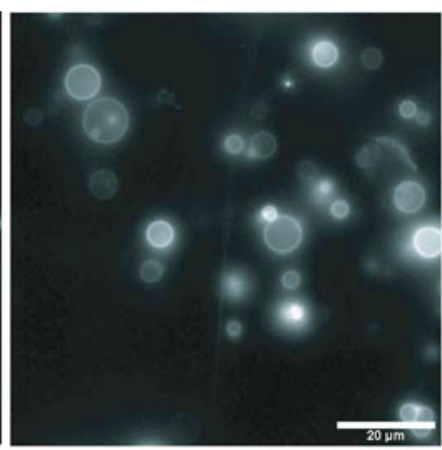

(c)

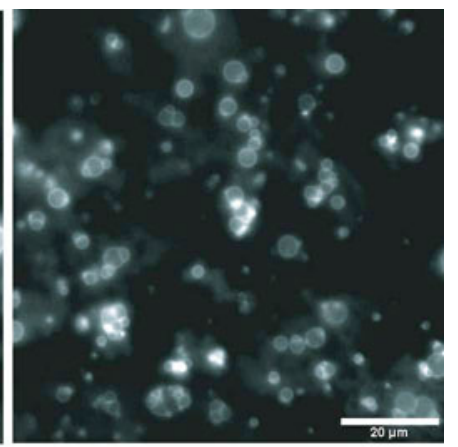

(d)

FIG. 4. (Color online) Fluorescent wide field microscopy images of negatively charged giant vesicles (DOPC/DOPE/CH/DPPG, 40/25/30/5 mol \%) immobilized on glass chamber slides (a) uncoated or coated with $1 \mathrm{mg} / \mathrm{ml}$, (b) native BSA, (c) cBSA-113, and (d) cBSA-147. Lipids were fluorescently stained with DiD. Bar scales to $20 \mu \mathrm{m}$.

tion behavior of SUV on different cBSA coatings on glass and polymer supports as well as the impact of varying charge densities on the vesicle fusion and lipid dynamics. Different BSA species (nBSA, cBSA-1113, and cBSA-147) coated on glass or polymer slides were probed with slightly negatively charged extruded lipid vesicles (DOPC/DPPG, 95/5 mol \%).

The SUV completely fused on uncoated hydrophilic glass forming a homogeneous fluid lipid bilayer, indicated by a high lateral diffusion mobility of DiD $\left(t_{1 / 2}=4.9 \pm 0.8 \mathrm{~s}\right)$ as shown in Fig. 3(a) and Table I, whereas a presumably homogeneous lipid monolayer was formed on the uncoated hydrophobic polymer slide [Fig. 3(e)] shown by hindered diffusion mobility of DiD $\left(t_{1 / 2}=14.6 \pm 0.8 \mathrm{~s}\right)$. The native BSA coating on the glass or polymer support nearly prevented the fusion or attachment of SUV as shown in Figs. 3(b) and 3(f). Only a few lipid patches with a surface coverage less than $20 \%$ and no immobilized SUVs were observed.

In contrast, the cBSA coatings on the polymer or glass support led to the immobilization of SUV and the formation of a lipid film [Figs. 3(c), 3(d), 3(g), and 3(h)]. The lipid bilayer on cBSA coated glass was patterned, whereas the lipid bilayer was more homogeneous on the polymer support and generally populated with a higher number of SUVs. Lateral diffusion mobility of the lipid tracer DiD in the lipid bilayer on cBSA coated glass was $\sim 2$ and fourfold restricted for cBSA-113 $\left(t_{1 / 2}=12.5 \pm 4.1 \mathrm{~s}\right)$ and cBSA-147 $\left(t_{1 / 2}\right.$ $=13.1 \pm 1 \mathrm{~s}$ ), respectively, compared within the lipid bilayer on bare glass. A similar decrease in lateral lipid mobility was observed on the cBSA coated polymer (Table I). The effect of the cBSA coating on the lateral lipid mobility was also observed in terms of $\sim 10 \%-30 \%$ decreased mobile fractions (Table I). The decreased lipid tracer mobility could be due to mixed adsorption of fused and adherent vesicles and due to electrostatic interactions between the oppositely charged protein-lipid surfaces.

Käsbauer et al. investigated whether the charged lipid constituents of a supported bilayer are symmetrically distributed between its two leaflets or if the solid surface charge gives rise to static lipid asymmetries. ${ }^{42}$ They observed for positively charged lipids a significant asymmetry between the distal and the proximal monolayer of a supported bilayer after $12 \mathrm{~h}$ incubation at low ionic strength $(20 \mathrm{mM} \mathrm{NaCl})$ by attenuated total reflection-infrared spectroscopy and NMR measurements. In order to pay attention to a possible rearrangement of negatively charged lipids into the proximal layer, we repeated the FRAP measurements 24 and $72 \mathrm{~h}$ after surface preparation.

\section{Immobilization of GUVs on cBSA coated polymer and glass slides}

Zwitterionic (DOPC/DOPE/CH, 45/25/30 mol \%) or negatively charged GUVs (DOPC/DOPE/CH/DPPG, $40 / 25 / 30 / 5 \mathrm{~mol} \%, 10 \mathrm{mM}$ ) filled with sucrose were immobilized on the BSA modified hydrophobic polymer or glass slides. The vesicles were diluted in salt buffer and incubated for a short time $(\sim 15 \mathrm{~min})$ on the surface, where they sedimented on the modified surface due to a density difference between the buffer outside and sucrose inside the vesicle. Unbound vesicles were removed by rinsing with PBS. There was a significant difference in the adsorption behavior of the GUVs depending on the type of BSA: before rinsing with PBS, similar amounts of GUVs attached onto the nBSA and cBSA coated surfaces, but the vesicles adhering to the nBSA-treated surface showed a dynamic behavior and a certain mobility, whereas the vesicles coated onto the cBSA treated surfaces were completely static (see Ref. 37, movies 1 and 2). After rinsing, higher numbers of GUVs stayed attached to the cBSA surfaces. In Figs. 4 and 5, representative fluorescence images of immobilized GUVs on glass or polymer chamber slides for different BSA coatings are shown. Giant vesicles tend to fuse on uncoated glass slides [Fig. 4(a)]. In contrast, the GUVs remain intact on uncoated COP/ COC polymer slides [Fig. 5(a)] and the spherical shape was better preserved when compared with immobilized GUVs on cBSA coating [Fig. 5(b)]. However, the giant vesicles immobilized on cBSA provided a higher stability against mechanical forces such as washing or liquid flow. The immobilized vesicles on cBSA stayed stable for more than 5 weeks (Fig. 10 of Ref. 37). Fluorescence microscopy and bleaching experiments revealed that some vesicles fused on the coated as 


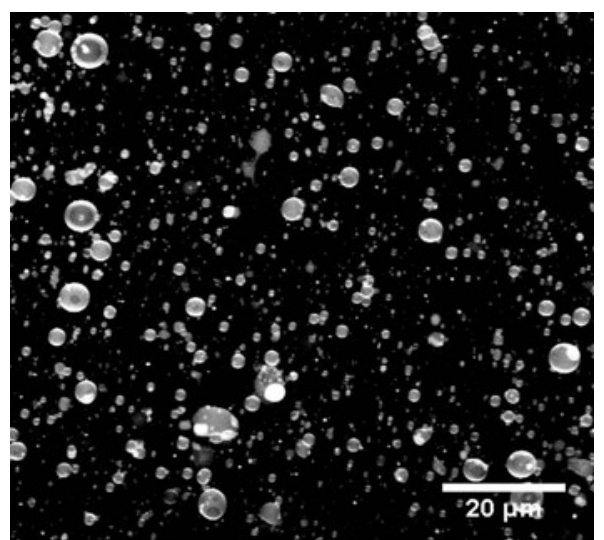

(a)

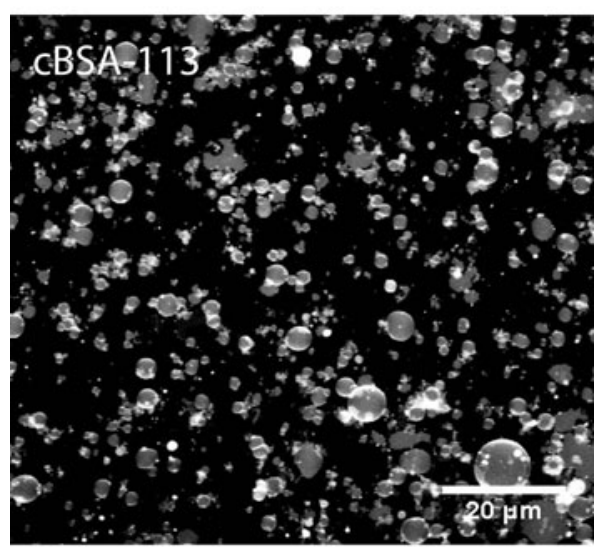

(b)

FIG. 5. Fluorescent confocal microscopy images of negatively charged giant vesicles (DOPC/DOPE/CH/DPPG, 40/25/30/5 mol \%) immobilized on a hydrophobic polymer chamber slide (a) without and (b) with cBSA-113 coating after rinsing with PBS buffer.

well as on the uncoated polymer surface (Fig. 6), forming probably a slightly negatively charged repelling lipid layer, which prevents further vesicle fusion and stabilizes the giant vesicles. Figure 7 sketches a model of lipid vesicle immobilization by cBSA coating on different supports.

To analyze if the method is limited to anionic liposomes and how much negative charge is necessary for the GUV immobilization, we prepared GUVs of zwitterionic DOPC or DOPC vesicles with increasing amounts of anionic DPPG (5\%-30\%). Phase contrast microscopy revealed that pure DOPC vesicles also attached to the cationized BSA $(-113$, -147). After rinsing with PBS, less zwitterionic GUVs remained immobilized on the surface compared with negatively charged GUVs $(5-10 \mathrm{~mol} \%)$. The pure DOPC vesicles also showed a strong tendency to form multiliposome aggregates, where only one or two liposomes attach to the surface and the others were faced to the bulk buffer phase. Stable immobilization of negatively charged GUVs was obtained for a 5-10 mol \% DPPG binary lipid mixture with DOPC. GUVs with 20-30 mol \% negatively charged DPPG almost completely fused on the cBSA as we observed by fluorescent staining with the lipophilic dye DiD (Fig. 11 of Ref. 37).
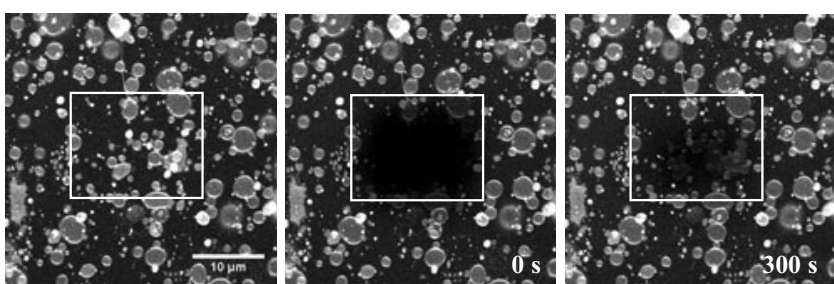

(a)
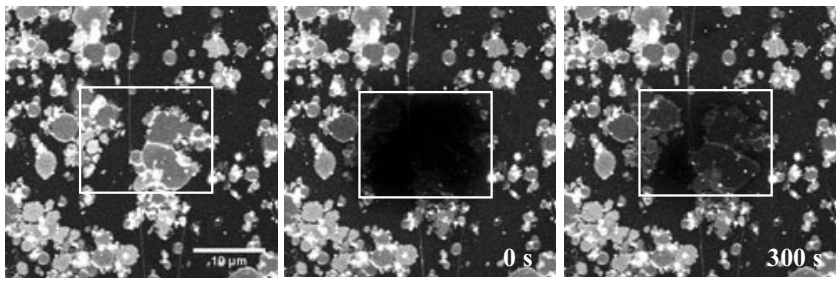

(b)

FIG. 6. Prebleached (left) and postbleached $(0,300 \mathrm{~s})$ fluorescent confocal microscopy images of negatively charged giant vesicles (DOPC/DOPE/CH/ DPPG, 40/25/30/5 mol \%) immobilized on a hydrophobic polymer chamber slide (a) without and (b) with cBSA-113 coating. The GUVs were stained with the lipophilic fluorescent dye DiD (1.5 nM).

\section{SUMMARY AND CONCLUSIONS}

We have studied the adsorption of cationized BSA on glass, polymer (COP/COC), and gold surfaces as a universal coating for the formation of electrostatically tethered lipid bilayers and the concomitant immobilization of lipid vesicles. SPS analysis showed high surface coverage and monolayer formation of cBSA species on gold, which was strongly dependent on concentration, indicating a dependency between higher protein concentration and aggregation resulting in lower surface coverage [Figs. 2(a) and 2(b)]. The application of cBSA-113 and cBSA-147 with different charge densities has an impact on the lateral diffusion of lipid bilayers on glass. In this way, manipulating or finetuning vesicle dynamics by changing the polyelectrolyte surface coating and ionic strength of the buffer is feasible and will be studied in the future. The number of immobilized vesicles appeared similar on cBSA coated polymer and glass surfaces. The cationized BSA coating influenced the net charge effectively from negative to positive surface potential [Fig. 1(b)], offering an easy to handle, fast $(\sim 1 \mathrm{~h})$, stable ( $\sim 5$ weeks), and economic polycationic surface for the immobilization of zwitterionic and negatively charged lipid vesicles. The cBSA coated surfaces are especially suitable

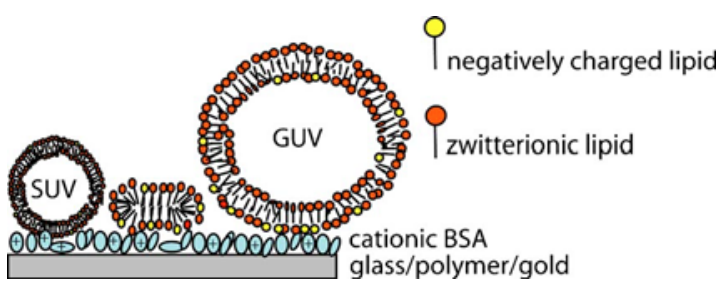

FIG. 7. (Color online) Schema of liposome immobilization on cationized BSA coated supports. 
for the immobilization of giant lipid vesicles, thus enabling future studies in the field of membrane-related materials and processes.

\section{ACKNOWLEDGMENTS}

The authors like to thank Kaloian Koynov and Andreas Best (MPI-P) for the helpful discussion about FRAP analysis and Günter Jansen (Beckman Coulter) for the technical instruction into the Delsa NanoC. Financial support by the NUS start-up grant under Grant No. WBS-R143-000-381133 and the SFB 625 is gratefully acknowledged.

${ }^{1}$ D. M. Rosenbaum, S. G. F. Rasmussen, and B. K. Kobilka, Nature (London) 459, 356 (2009).

${ }^{2}$ M. Majdi and H.-S. V. Chen, Recep. Ligand Channel Res. 2, 59 (2009). ${ }^{3}$ P. Walde and S. Ichikawa, Biomol. Eng. 18, 143 (2001).

${ }^{4}$ R. Labas, F. Beilvert, B. Barteau, S. David, R. Chevre, and B. Pitard, Genetica (Dordrecht, Neth.) 138, 153 (2009).

${ }^{5}$ G. Cevc, Adv. Drug Delivery Rev. 56, 675 (2004).

${ }^{6}$ L. F. Zhang, L. Hong, Y. Yu, S. C. Bae, and S. Granick, J. Am. Chem. Soc. 128, 9026 (2006).

${ }^{7}$ M. L. Moraes, M. S. Baptista, R. Itri, V. Zucolotto, and O. N. Oliveira, Jr., Mater. Sci. Eng., C 28, 467 (2008).

${ }^{8}$ P. Lundahl and Q. Yang, J. Chromatogr. 544, 283 (1991).

${ }^{9}$ L. S. Jung, J. S. Shumaker-Parry, C. T. Campbell, S. S. Yee, and M. H. Gelb, J. Am. Chem. Soc. 122, 4177 (2000).

${ }^{10}$ L. Masson, A. Mazza, and R. Brousseau, Anal. Biochem. 218, 405 (1994).

${ }^{11}$ S. Svedhem, I. Pfeiffer, C. Larsson, C. Wingren, C. Borrebaeck, and F. Hook, ChemBioChem 4, 339 (2003).

${ }^{12}$ C. Yoshina-Ishii and S. G. Boxer, J. Am. Chem. Soc. 125, 3696 (2003).

${ }^{13}$ T. Stora, Z. Dienes, H. Vogel, and C. Duschl, Langmuir 16, 5471 (2000).

${ }^{14}$ S. Schuy, B. Treutlein, A. Pietuch, and A. Janshoff, Small 4, 970 (2008).

${ }^{15}$ G. Klenkar, B. Brian, T. Ederth, G. Stengel, F. Hook, J. Piehler, and B. Liedberg, BioInterphases 3, 29 (2008).

${ }^{16}$ S. M. Christensen and D. Stamou, Soft Matter 3, 828 (2007).

${ }^{17}$ D. Merkle, N. Kahya, and P. Schwille, ChemBioChem 9, 2673 (2008).

${ }^{18}$ B. G. Lorz, A. S. Smith, C. Gege, and E. Sackmann, Langmuir 23, 12293 (2007).

${ }^{19}$ D. Volodkin, V. Ball, P. Schaaf, J. C. Voegel, and H. Mohwald, Biochim. Biophys. Acta 1768, 280 (2007).

${ }^{20}$ A. L. Bernard, M. A. Guedeau-Boudeville, L. Jullien, and J. M. di Meglio, Langmuir 16, 6809 (2000).

${ }^{21}$ J. O. Rädler, T. J. Feder, H. H. Strey, and E. Sackmann, Phys. Rev. E 51, 4526 (1995).

${ }^{22}$ C. H. Reccius and P. Fromherz, Langmuir 20, 11175 (2004).

${ }^{23}$ X. Liu, R. Zhao, Y. Zhang, X. Jiang, J. Yue, P. Jiang, and Z. Zhang,
Biochim. Biophys. Acta 1770, 1620 (2007).

${ }^{24}$ L. Zöphel, K. Eisele, R. Gropeanu, A. Rouhanipour, K. Koynov, I. Lieberwirth, K. Müllen, and T. Weil, Macromol. Chem. Phys. 211, 146 (2010).

${ }^{25}$ J. F. Ng, S. Jaenicke, K. Eisele, J. Dorn, and T. Weil, "Cationized albumin-biocoatings for the efficient chiral reduction in a microchannel reactor," BioInterphases (submitted).

${ }^{26}$ M. I. Angelova and D. S. Dimitrov, Faraday Discuss. Chem. Soc. 81, 303 (1986).

${ }^{27}$ R. Dimova, S. Aranda, N. Bezlyepkina, V. Nikolov, K. A. Riske, and R. Lipowsky, J. Phys.: Condens. Matter 18, S1151 (2006).

${ }^{28}$ E. K. Sinner, U. Reuning, F. N. Kok, B. Sacca, L. Moroder, W. Knoll, and D. Oesterhelt, Anal. Biochem. 333, 216 (2004).

${ }^{29}$ A. Sze, D. Erickson, L. Q. Ren, and D. Q. Li, J. Colloid Interface Sci. 261, 402 (2003).

${ }^{30}$ Y. L. Jeyachandran, J. A. Mielczarski, E. Mielczarski, and B. Rai, J. Colloid Interface Sci. 341, 136 (2010).

${ }^{31}$ N. Watanabe, T. Shirakawa, M. Iwahashi, K. Ohbu, and T. Seimiya, Colloid Polym. Sci. 264, 903 (1986).

${ }^{32} \mathrm{~W}$. Grant and R. Dehl, Adhesion and Adsorption of Polymers (Plenum Press, New York, 1980), p. 827.

${ }^{33}$ T. W. Xu, R. Q. Fu, and L. F. Yan, J. Colloid Interface Sci. 262, 342 (2003).

${ }^{34}$ K. Rezwan, L. P. Meier, M. Rezwan, J. Voros, M. Textor, and L. J. Gauckler, Langmuir 20, 10055 (2004).

${ }^{35} \mathrm{H}$. Larsericsdotter, S. Oscarsson, and J. Buijs, J. Colloid Interface Sci. 289, 26 (2005).

${ }^{36} \mathrm{~K}$. Eisele et al., Biomaterials 31, 8789 (2010).

${ }^{37}$ See supplementary material at E-BJIOBN-5-011003 for additional information about the zeta potential of adsorbed BSA and cBSA on glass demonstrating that the positive charge effect of cationized BSA is even stronger in water than in DPBS (Fig. 8). Fluorescence images of fluorescent labelled cBSA and the corresponding phase contrast images of adsorbed GUVs showed that the cBSA layer is intact after vesicle immobilization (Fig. 9). Long time stability of immobilized GUVs (DOPC/ DPPG) on cBSA-113 was observed for GUVs with 5-10 mol\% negatively charged DPPG [Figs. 10(a) and 10(b)], whereas pure DOPC vesicles were less stable [Fig. 10(a)]. Increasing the concentration of negatively charged DPPG $(0,5,10,20,30 \%)$ in GUVs also resulted in stronger surface interaction with the positively charged cBSA-113 and vesicle fusion. The document may also be reached via the EPAPS homepage (http:// www.aip.org/pubservs/epaps.html) or from ftp.aip.org in the directory /epaps/. See the EPAPS homepage for more information.

${ }^{38}$ S. H. Behrens and D. G. Grier, J. Chem. Phys. 115, 6716 (2001).

${ }^{39} \mathrm{~V}$. Kahl, ibidi GmbH, Martinsried, Germany (private communication).

${ }^{40}$ S. Brantzen, F. Volklein, W. Knoll, and B. Menges, Sens. Actuators, A 135, 492 (2007).

${ }^{41}$ G. T. Hermanson, Bioconjugate Techniques (Academic, New York, 1996), Vol. 27, p. 785 .

${ }^{42}$ M. Käsbauer, M. Junglas, and T. M. Bayerl, Biophys. J. 76, 2600 (1999). 